\title{
Mathematical modeling of multi-drugs therapy: a challenge for determining the optimal combinations of antiviral drugs
}

Yoshiki Koizumi ${ }^{1}$ and Shingo Iwami $i^{*^{*}}$

\author{
* Correspondence: \\ siwami@kyushu-u.org \\ ${ }^{2}$ Department of Biology, Faculty of \\ Sciences, Kyushu University, 6-10-1 \\ Hakozaki, Higashi-ku, Fukuoka \\ 812-8581, Japan \\ Full list of author information is \\ available at the end of the article
}

\begin{abstract}
In the current era of antiviral drug therapy, combining multiple drugs is a primary approach for improving antiviral effects, reducing the doses of individual drugs, relieving the side effects of strong antiviral drugs, and preventing the emergence of drug-resistant viruses. Although a variety of new drugs have been developed for HIV, HCV and influenza virus, the optimal combinations of multiple drugs are incompletely understood. To optimize the benefits of multi-drugs combinations, we must investigate the interactions between the combined drugs and their target viruses. Mathematical models of viral infection dynamics provide an ideal tool for this purpose. Additionally, whether drug combinations computed by these models are synergistic can be assessed by two prominent drug combination theories, Loewe additivity and Bliss independence. By combining the mathematical modeling of virus dynamics with drug combination theories, we could show the principles by which drug combinations yield a synergistic effect. Here, we describe the theoretical aspects of multi-drugs therapy and discuss their application to antiviral research.
\end{abstract}

Keywords: Antiviral therapy, Mathematical modeling, Drug combination theory, Virus dynamics

\section{Background}

Several landmark mathematical modeling studies of anti-retroviral therapy were reported in 1995 [1-4]. Thereafter, mathematical modeling has provided quantitative insights into antiviral drugs targeting HIV [5-13], HCV [14-18], HBV [19,20] and influenza virus [21,22]. These studies have elucidated the key steps of viral replication cycles and viral infection dynamics, such as the half-life of viruses and how viruses replicate in cells (reviewed in [23-27]). Although mathematical viral models could also search for drug combinations that improve antiviral effects and prevent the emergence of drug-resistant viruses, this potential of viral modeling has yet to be properly explored. Multi-drug administration is a standard treatment for HIV and HCV infection, and is also required in HBV and influenza. The established combination drug therapy for HIV, known as highly active anti-retroviral therapy (HAART), remains prohibitively expensive in developing countries. Additionally, HIV patients receiving long-term HAART may experience severe side effects such as lipodystrophy, hepatotoxicity, renal dysfunction, peripheral neuropathy and cardiovascular diseases (reviewed in [28]). By

(c) 2014 Koizumi and Iwami; licensee BioMed Central Ltd. This is an Open Access article distributed under the terms of the Creative Commons Attribution License (http://creativecommons.org/licenses/by/4.0), which permits unrestricted use, distribution, and reproduction in any medium, provided the original work is properly credited. The Creative Commons Public Domain Dedication waiver (http://creativecommons.org/publicdomain/zero/1.0/) applies to the data made available in this article, unless otherwise stated. 
optimizing drug combinations, we could reduce the doses of individual drugs, thereby lowering the cost of treatment, reducing side effects, enhancing the antiviral effects and reducing the risk of drug-resistant viruses.

To accelerate the above benefits of drug combinations, we need to know whether or not a drug combination exerts a synergistic effect (reviewed in [29,30]). A nonsynergistic drug combination requires a larger than expected dose to achieve a regular antiviral effect. Although multi-drug therapy is standard practice, the complexity of the combined drugs' responses is improperly understood, and rendered counter-intuitive by drug absorption, distribution, metabolism, and excretion through drug transporters [28]. To overcome these difficulties, comprehensive trials (or experiments) of various combinations and doses of drugs have been required. The combined effect has been investigated by empirical methods such as Loewe additivity [29,31] and Bliss independence $[29,32]$. Both theories have evolved from pharmacological research, and are used to classify combination effects as synergistic, additive or antagonistic [29,33,34]. These empirical frameworks have become widely accepted in pharmacology and have provided quantitative insight into drug combinations. However, because their underlying mechanics are unknown, the frameworks cannot provide reasons for a given combination effect (e.g. why is this drug combination additive, whereas that combination is antagonistic?). Showing the mechanisms of drug combination effects is expected to remarkably advance the development of multicomponent therapeutics. Thus, in addition to judging drug combination effects, knowledge of the mechanism is crucial to fundamental antiviral research.

Here we discuss two primary concepts of drug combination theory: Loewe additivity and Bliss independence. We apply these models to viral replication in a host cell, and discuss their utility for understanding two-drug interactions and viral dynamics. Finally, we discuss how drug combination theory might be applied to cancer chemotherapies and antiviral therapies. Combining mathematical models of viral replication with drug combination theory, we can establish an efficient framework for optimizing drug combinations.

\section{Basic drug combination theory: Loewe additivity}

Loewe additivity is regarded as a primary criterion for evaluating drug combination effects [29]. To conceptualize Loewe additivity, let us consider the simplest situation, in which a combination of drugs A and B has a synergistic, additive or antagonistic effect. If the effect is additive, the Loewe additivity is defined as [31]:

$$
\frac{C_{A}^{*}}{C_{A}}+\frac{C_{B}^{*}}{C_{B}}=1
$$

where $c_{A}^{*}$ and $c_{B}^{*}$ are the concentrations of drugs $\mathrm{A}$ and $\mathrm{B}$ respectively in the combined dose, and $C_{A}$ and $C_{B}$ are the respective concentrations of drugs $\mathrm{A}$ and $\mathrm{B}$ that produce the same effect as the drug combination. That is, the Loewe additivity specifies the concentration ratio of a single drug and its combination with another drug. Note that Loewe additivity assumes that two drugs target the same molecule or pathway. If 
two drugs do not mutually interact, they can be related through the combination index

$(\mathrm{CI})$ based on the mass action law derived by Chou and Talalay [35]:

$$
C I=\frac{C_{A}^{*}}{C_{A}}+\frac{C_{B}^{*}}{C_{B}}
$$

where the right hand side of Eq. (2) is identical to the left side of Eq. (1). When $\mathrm{CI}<1$, the relationship is synergetic, when $\mathrm{CI}=1$, it is additive, and when $\mathrm{CI}>1$, it is antagonistic. For example, suppose that $4 \mu \mathrm{M}$ of drug $\mathrm{A}$ and $5 \mu \mathrm{M}$ of drug B exert the same effect as a combination of $1 \mu \mathrm{M}$ of A and $2 \mu \mathrm{M}$ of B. Substituting these concentrations into Eq. (2), we obtain $C I=0.65(=1 / 4+2 / 5<1)$, implying that the drug combination is synergistic. Note that the $C I$ does not express the extent of synergy or antagonism; it is merely a criterion that separates the two behaviors without quantifying them.

To evaluate a drug combination effect by the $C I$, we require dose-response curves of drugs $\mathrm{A}$ and $\mathrm{B}$, from which we can determine $c_{A}^{*}$ and $c_{B}^{*}$. The effect of a single drug $E$ is modeled by the Hill function as follows:

$$
E=E_{\max } \frac{c^{h}}{c^{h}+I C_{50}^{h}}
$$

where $E_{\max }$ is the maximum effect, $c$ is the drug concentration, $h$ is the Hill coefficient that determines the steepness of the dose-response curve, and $I C_{50}$ is the concentration at which $E$ exerts $50 \%$ of its maximum effect. Substituting Eq. (3) into Eq. (1) and rearranging in the case of $E_{\max }=1$, we obtain:

$$
\frac{C_{A}^{*}}{I C_{50_{A}}\left(\frac{E}{1-E}\right)^{\frac{1}{h_{A}}}}+\frac{C_{B}^{*}}{I C_{50_{B}}\left(\frac{E}{1-E}\right)^{\frac{1}{h_{B}}}}=1 .
$$

Numerically solving Eq. (4) for $E$, we can predict the additive effect at any drug concentrations $c_{A}^{*}$ and $c_{B}^{*}[35,36]$. The following section explains an example of applying Loewe additivity to estimate rational drug combinations and optimal doses.

\section{Application of Loewe additivity for estimating rational drug combinations and optimal doses}

We here explain the usefulness of combining the Loewe additivity with mathematical modeling of hepatitis $\mathrm{C}$ virus (HCV) replication. The aim is to optimize the antiviral drug combination. Combining multiple drugs such as interferon- $\alpha$ and ribavirin is a standard therapy for enhancing the antiviral effects and reducing the risk of drugresistant viruses. However, the conventional anti-HCV drugs fail to eradicate $\mathrm{HCV}$ genotype 1 in about $40 \%$ patients and may cause severe side effects. To overcome these problems, researchers have developed and administered direct acting antiviral (DAA) drugs that target viral-specific proteins such as viral proteases, as adjuncts to conventional anti-HCV drugs. To understand the principle behind optimal drug combinations and doses, we focused on the essential features of $\mathrm{HCV}$ replication and the mechanisms of the antiviral drugs (Figure 1A). Using previous mathematical models of HCV replication in a host cell [37-40], a modified mathematical model accounting for the mechanisms 


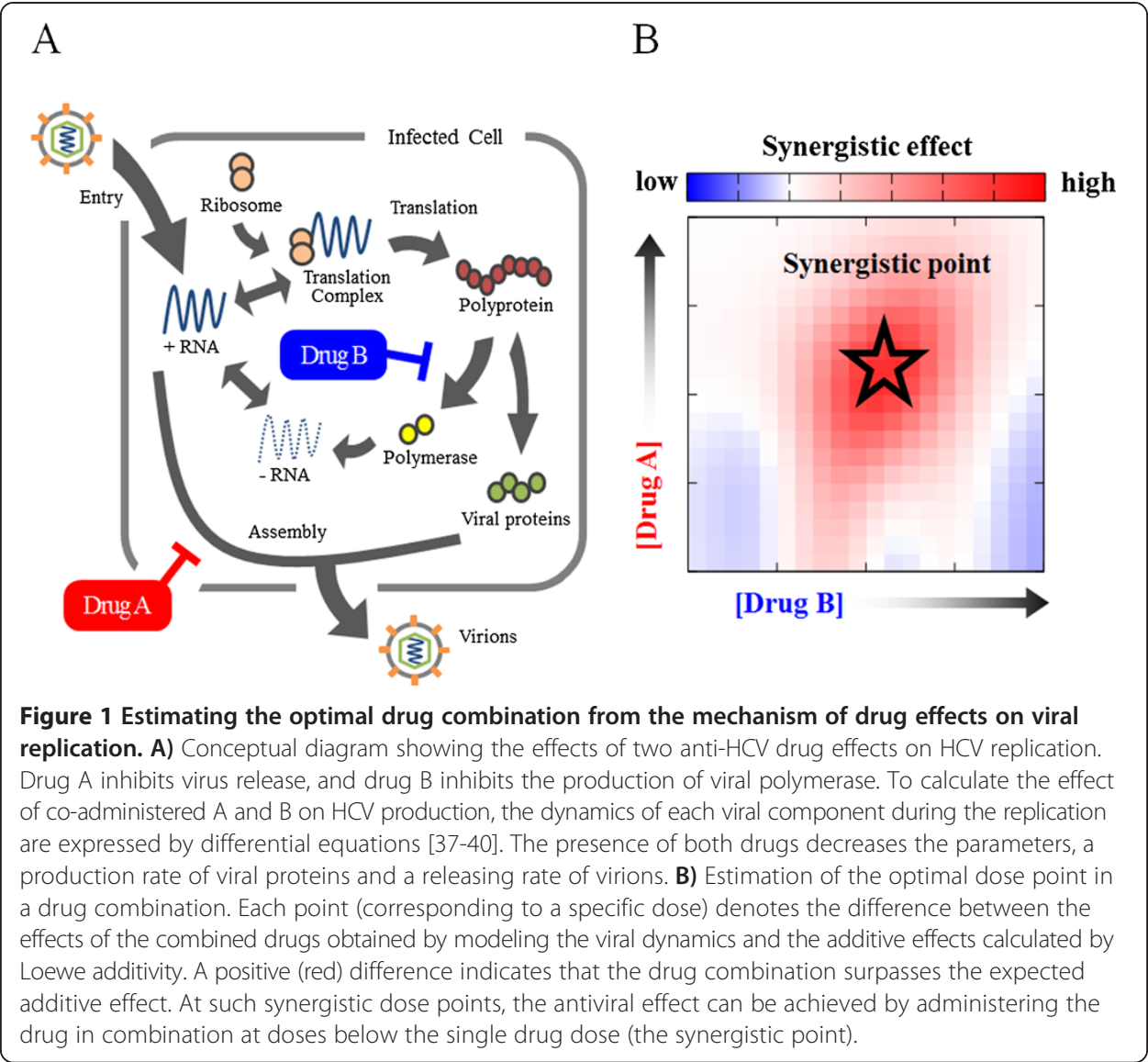

of drug actions would quantitatively simulate the anti-HCV effects at any dose of a drug combination. The effects of the drug combination at specific doses could then be evaluated by the Loewe additivity. In this way, we could estimate the synergistic dose point of a drug combination that enhances the anti-HCV effects (Figure 1B). The above analysis could predict the optimal drug combination that both reduces the total dose of each drug and enhances their anti-HCV effects.

\section{The alternative drug combination theory: Bliss independence}

Bliss independence is the other major criterion for clarifying drug combination effects [32]. Bliss independence defines the expectation of a combined drug effect, calculated by multiplying the probabilities of the individual drugs. It assumes that each drug independently targets a different stage of viral replication via a different mechanism, with no interaction between the drug actions. Bliss independence expresses the unaffected probability of a two-drug combination $U_{A B}$ as the product of the unaffected probabilities $U_{A}$ and $U_{B}$ of drugs $\mathrm{A}$ and $\mathrm{B}$ :

$$
U_{A B}=U_{A} \times U_{B}
$$

Intuitively, Eq. (5) implies that targets bypassed by drug A will be intercepted by drug B. The total probability of missed targets is obtained by multiplying the probabilities of 
the unaffected targets. Substituting $U$ by $1-P$, where $P$ denotes the probability of an intercepted target, Eq. (5) can be expressed as $\left(1-P_{A B}\right)=\left(1-P_{A}\right) \times\left(1-P_{B}\right)$, or

$$
P_{A B}=P_{A}+P_{B}-P_{A} P_{B} .
$$

If the combined effect of the single drug doses is consistent with Eq. (6), this drug combination is evaluated as additive according to the Bliss independence measure. For instance, suppose that drugs A and B exhibit 50\% and 60\% inhibition, respectively. The Bliss independence predicts that drugs $\mathrm{A}$ and $\mathrm{B}$ will exert a combined inhibitory effect of $80 \%$, calculated as $P_{A B}=0.5+0.6-0.5 \times 0.6=0.8$.

Unlike the Loewe additivity, the Bliss independence can evaluate the effect of a drug combination without requiring dose-response curves of the single drugs. If we attempt to assess a drug combination by the Loewe additivity, we must measure the effects at several single doses for constructing the dose-response curve. In contrast, the Bliss independence can assess the effects of a drug combination from a single point. Thus, the Bliss independence is useful if experimental data are limited. Additionally, in specific cases, the Bliss independence and Loewe additivity can be rendered equivalent using the Hill function Eq. (3) [41]. The following section explains an example of applying Bliss independence to quantifying the antiviral effects of intrinsic factors on HIV replication.

\section{Quantifying the antiviral effects of APOBEC3G on HIV replication by Bliss independence}

The Bliss independence measure can predict antiviral effects as well as evaluate drug combinations. To illustrate this idea, we estimate the anti-HIV effect of a well-known antiviral host factor by Bliss independence [42]. Apolipoprotein B mRNA editing enzyme catalytic polypeptide-like 3G (APOBEC3G) suppresses HIV replication in cells by two main mechanisms: (1) inhibiting viral reverse transcriptase and (2) mutating cytidine $(\mathrm{C})$ to uracil $(\mathrm{U})$ in viral DNA by cytidine deaminase activity (Figure 2A). Although the anti-HIV effect of reverse transcriptase inhibition can be measured using a mutated APOBEC3G (with its C-to- $\mathrm{U}$ activity removed) (Figure 2A), the extent to which the mutation itself inhibits HIV replication cannot be experimentally determined. To quantify the anti-HIV effect of C-to-U mutation by APOBEC 3G, we can use Bliss independence to model the unaffected fractions of viral infectivity of wildtype (WT) and mutant APOBEC3G, denoted $f_{W T}$ and $f_{C I}$ respectively, in terms of the unaffected fractions of viral infectivity by reverse transcription inhibition and C-to- $\mathrm{U}$ mutation, $f_{R T}$ and $f_{M u}$, as follows:

$$
f_{W T}(x)=f_{R T}(x) \times f_{M u}(x)=\left(1-\frac{x^{h_{R T}}}{x^{h_{R T}}+I C_{50_{R T}}^{h_{R T}}}\right) \times\left(1-\frac{x^{h_{M u}}}{x^{h_{M u}}+I C_{50_{M u}}^{h_{M u}}}\right),
$$

and

$$
f_{C I}(x)=f_{R T}(x)=\left(1-\frac{x^{h_{R T}}}{x^{h_{R T}}+I C_{50_{R T}}^{h_{R T}}}\right),
$$

where $x$ is the expression level of APOBEC3G, $h_{R T}$ and $h_{M u}$ are Hill coefficients, and $I C_{50_{R T}}$ and $I C_{50_{M u}}$ are the expression levels required to achieve $50 \%$ inhibition by reverse transcription inhibition and $\mathrm{C}$-to- $\mathrm{U}$ mutation, respectively. We estimated 4 


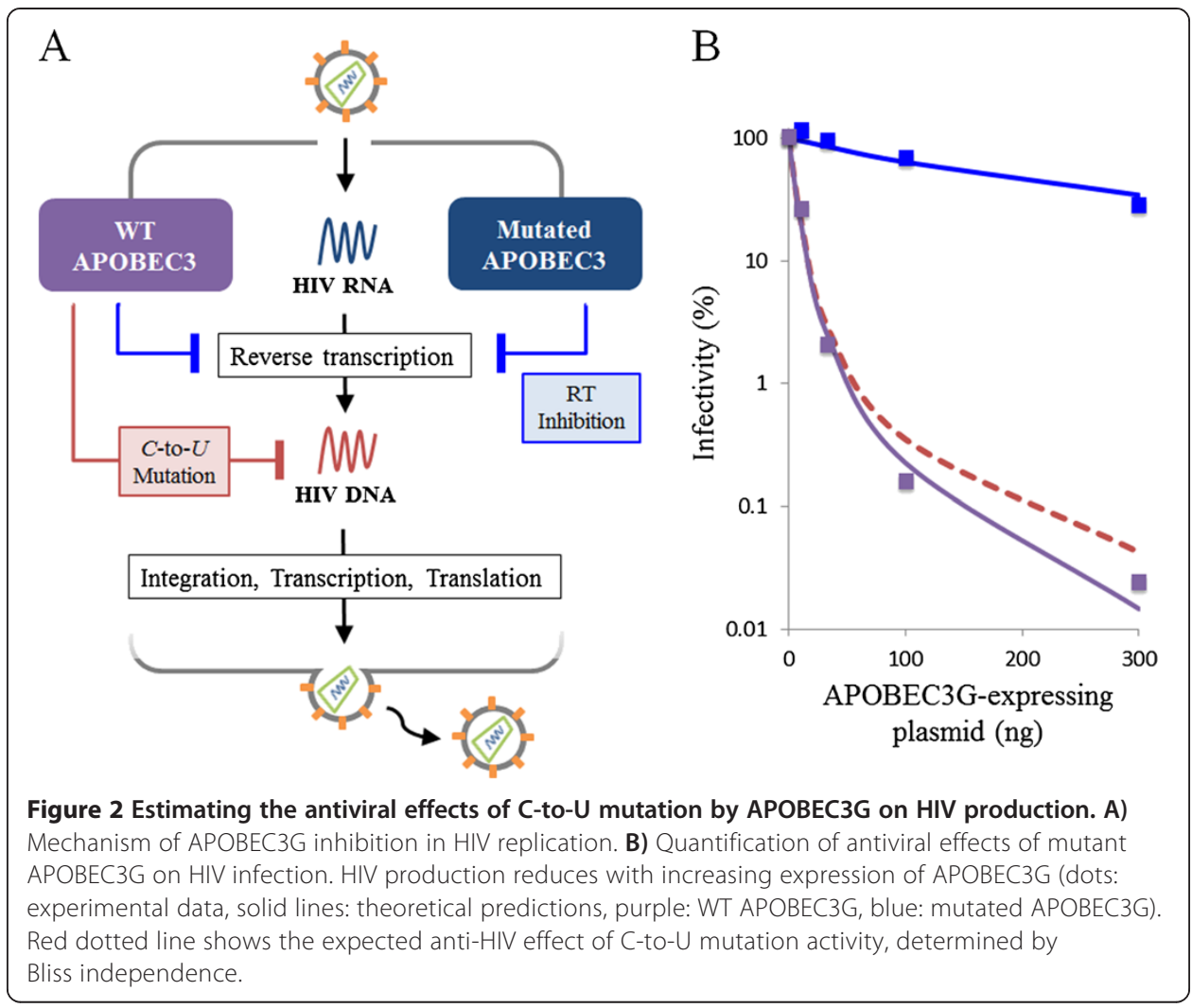

parameters $\left(h_{R T}, h_{M u}, I C_{50_{R T}}\right.$, and $\left.I C_{50_{M u}}\right)$ by fitting to experimental data of WT and mutated APOBEC3G (Figure 2B). Thus, the anti-HIV effect of C-to-U mutation by APOBEC3G can be quantitatively estimated by a Bliss independence-based modeling approach.

\section{Potential applications of drug combination theory}

The Loewe additivity and Bliss independence form the basis of sophisticated protocols for assessing drug combinations, especially in cancer-targeted chemotherapy [33,34]. Research on biological network systems, as well as the biochemical characteristics of drugs, has also unraveled the mechanisms by which drug combinations produce synergistic effects (reviewed in [41,43-45]). Such research is important because (1) it facilitates rational drug discovery and (2) it predicts unknown connectivities in biological networks. Regarding the first point, the effects of a developing drug combined with conventional drugs are maximized by investigating biological networks, and rational drug targets are decided. For example, using computational modeling of cancer signaling networks and animal experiments, Kirouac et al. [46] identified the optimal inhibitor combination for suppressing the growth of ERBB2-amplified breast cancer. They adopted Bliss independence as a synergy criterion. Supplementing this computational approach with experimental data is useful for developing theoretically effective novel drugs. Regarding point (2), the biological networks of signaling pathways constructed by various components can be predicted from the dose-response shapes of drug combinations (reviewed in [47]). Lehar et al. [48] used several drug combination criteria, including Loewe additivity and Bliss independence, to connect biological 
components from the results of cellular responses to chemical combinations. This approach assumes that the dose-response shape of a drug combination depends on how drug targets are connected in a biological system.

The above research potentially benefits not only cancer chemotherapy, but also antiviral therapy development. For instance, Owens et al. [49] showed how HCV replication responds to chemical combinations that inhibit some enzymes involved in the sterol biosynthesis pathway. Supplementing computational simulation with in vitro experiments, they identified a rational inhibitor combination as a novel drug candidate. Mathematical models of HCV dynamics during drug therapy have also been investigated at the intracellular level [18], the intercellular level [14-17,50,51], and on a scale encompassing both levels [51-54]. These studies have quantitatively elucidated the properties of anti-HCV drugs such as interferon and protease inhibitors. Furthermore, by inputting clinical data to a mathematical model of HCV dynamics, Rong et al. [55] derived a mechanism by which drug-resistant viruses can rapidly emerge during single-drug therapy. Applying drug combination theories to these studies, mathematical models of HCV dynamics could be used to optimize the anti-HCV drug dosage and combination, thereby enhancing the anti-viral effect and reducing the risk of drug-resistant viruses (Figure 3).

\section{Conclusion}

We have reviewed two major drug combination theories, Loewe additivity and Bliss independence, and discussed how combining these theories with mathematical modeling of viral dynamics might assist antiviral drug therapy. In addition, we have proposed an efficient framework for optimizing drug combinations and quantifying the anti-viral effect. Based on previous studies of computational virology, the integration of drug combination theory and dynamic modeling is a new approach with great potential for showing viral responses to drug combinations, and accelerating novel antiviral drug discovery.

\section{Integration of virus dynamics and drug combination theory}

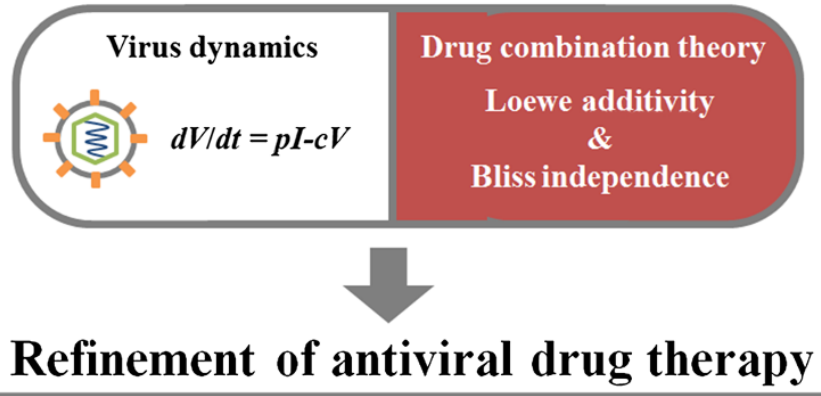

Prediction of a drug combination effects in vitro and in vivo. Estimation of the optimal drug combination and the doses. Elucidation of the mechanism of a drug combination.

Figure 3 Concept of integrating drug combination theory and viral dynamics. The effect of a drug combination calculated by mathematical modeling of viral dynamics could be assessed by the Loewe additivity and Bliss independence. This approach could improve antiviral therapy by predicting the effect of a drug combination in vivo and in vitro, estimating the optimal drug dosage and combination, and discovering how the drugs interact. 
Competing interests

The authors declare that they have no competing interests.

\section{Authors' contributions}

Conceived and designed the study: YK SI. Wrote the paper: YK SI. Both authors read and approved the final manuscript.

\section{Acknowledgments}

This research is partly supported by a Grants-in-Aid for Young Scientists B25800092 (to S.I.) from the Japan Society for the Promotion of Science (JSPS); the Kyushu University Interdisciplinary Programs in Education and Projects in Research Development (to S.I.); the Aihara Innovative Mathematical Modeling Project, JSPS through the "Funding Program for World-Leading Innovative R \& D on Science and Technology (FIRST program)", initiated by Council for Science and Technology Policy (to S.I.).

\section{Author details}

${ }^{1}$ School of Medicine, College of Medical, Pharmaceutical and Health Sciences, Kanazawa University, 13-1 Takaramachi, Kanazawa-shi, Ishikawa 920-8640, Japan. ${ }^{2}$ Department of Biology, Faculty of Sciences, Kyushu University, 6-10-1 Hakozaki, Higashi-ku, Fukuoka 812-8581, Japan.

Received: 7 June 2014 Accepted: 15 September 2014 Published: 25 September 2014

\section{References}

1. Ho DD, Neumann AU, Perelson AS, Chen W, Leonard JM, Markowitz M: Rapid turnover of plasma virions and CD4 lymphocytes in HIV-1 infection. Nature 1995, 373:123-126.

2. Wei X, Ghosh SK, Taylor ME, Johnson VA, Emini EA, Deutsch P, Lifson JD, Bonhoeffer S, Nowak MA, Hahn BH: Viral dynamics in human immunodeficiency virus type 1 infection. Nature 1995, 373:117-122.

3. Nowak MA, Bonhoeffer S, Loveday C, Balfe P, Semple M, Kaye S, Tenant-Flowers M, Tedder R: HIV results in the frame. Results confirmed. Nature 1995, 375:193.

4. Coffin JM: HIV population dynamics in vivo: implications for genetic variation, pathogenesis, and therapy. Science 1995, 267:483-489.

5. Perelson AS, Neumann AU, Markowitz M, Leonard JM, Ho DD: HIV-1 dynamics in vivo: virion clearance rate, infected cell life-span, and viral generation time. Science 1996, 271:1582-1586.

6. Wein LM, Zenios SA, Nowak MA: Dynamic multidrug therapies for HIV: a control theoretic approach. J Theor Biol 1997, 185:15-29.

7. Perelson AS, Essunger P, Cao Y, Vesanen M, Hurley A, Saksela K, Markowitz M, Ho DD: Decay characteristics of HIV-1-infected compartments during combination therapy. Nature 1997, 387:188-191.

8. Notermans DW, Goudsmit J, Danner SA, de Wolf F, Perelson AS, Mittler J: Rate of HIV-1 decline following antiretroviral therapy is related to viral load at baseline and drug regimen. AIDS 1998, 12:1483-1490.

9. Wein LM, D'Amato RM, Perelson AS: Mathematical analysis of antiretroviral therapy aimed at HIV-1 eradication or maintenance of low viral loads. J Theor Biol 1998, 192:81-98.

10. Di Mascio M, Markowitz M, Louie M, Hogan C, Hurley A, Chung C, Ho DD, Perelson AS: Viral blip dynamics during highly active antiretroviral therapy. J Virol 2003, 77:12165-12172.

11. Sedaghat AR, Dinoso JB, Shen L, Wilke CO, Siliciano RF: Decay dynamics of HIV-1 depend on the inhibited stages of the viral life cycle. Proc Natl Acad Sci U S A 2008, 105:4832-4837.

12. Shen L, Rabi SA, Sedaghat AR, Shan L, Lai J, Xing S, Siliciano RF: A critical subset model provides a conceptual basis for the high antiviral activity of major HIV drugs. Sci Trans/ Med 2011, 3:91ra63.

13. Rosenbloom DIS, Hill AL, Rabi SA, Siliciano RF, Nowak MA: Antiretroviral dynamics determines HIV evolution and predicts therapy outcome. Nat Med 2012, 18:1378-1385.

14. Zeuzem S, Schmidt JM, Lee JH, von Wagner M, Teuber G, Roth WK: Hepatitis C virus dynamics in vivo: effect of ribavirin and interferon alfa on viral turnover. Hepatology 1998, 28:245-252.

15. Neumann AU, Lam NP, Dahari H, Gretch DR, Wiley TE, Layden TJ, Perelson AS: Hepatitis C viral dynamics in vivo and the antiviral efficacy of interferon-alpha therapy. Science 1998, 282:103-107.

16. Dixit NM, Layden-Almer JE, Layden TJ, Perelson AS: Modelling how ribavirin improves interferon response rates in hepatitis C virus infection. Nature 2004, 432:922-924.

17. Dahari H, Ribeiro RM, Perelson AS: Triphasic decline of hepatitis C virus RNA during antiviral therapy. Hepatology 2007, 46:16-21.

18. Dahari H, Sainz B, Perelson AS, Uprichard SL: Modeling subgenomic hepatitis C virus RNA kinetics during treatment with alpha interferon. J Virol 2009, 83:6383-6390.

19. Lewin SR, Ribeiro RM, Walters T, Lau GK, Bowden S, Locarnini S, Perelson AS: Analysis of hepatitis B viral load decline under potent therapy: complex decay profiles observed. Hepatology 2001, 34:1012-1020.

20. Dahari H, Shudo E, Ribeiro RM, Perelson AS: Modeling complex decay profiles of hepatitis B virus during antiviral therapy. Hepatology 2009, 49:32-38.

21. Beauchemin CAA, MCSharry JJ, Drusano GL, Nguyen JT, Went GT, Ribeiro RM, Perelson AS: Modeling amantadine treatment of influenza A virus in vitro. J Theor Biol 2008, 254:439-451.

22. Dobrovolny HM, Gieschke R, Davies BE, Jumbe NL, Beauchemin CAA: Neuraminidase inhibitors for treatment of human and avian strain influenza: A comparative modeling study. J Theor Biol 2011, 269:234-244.

23. Nowak M, May RM: Virus Dynamics: Mathematical Principles of Immunology and Virology. USA: Oxford University Press; 2001:256.

24. Perelson AS: Modelling viral and immune system dynamics. Nat Rev Immunol 2002, 2:28-36.

25. Rong L, Perelson AS: Modeling HIV persistence, the latent reservoir, and viral blips. J Theor Biol 2009, 260:308-331. 
26. Guedj J, Rong L, Dahari H, Perelson AS: A perspective on modelling hepatitis C virus infection. J Viral Hepat 2010, 17:825-833.

27. Chatterjee A, Guedj J, Perelson AS: Mathematical modelling of HCV infection: what can it teach us in the era of direct-acting antiviral agents? Antivir Ther 2012, 17:1171-1182.

28. Hawkins T: Understanding and managing the adverse effects of antiretroviral therapy. Antiviral Res 2010, 85:201-209.

29. Greco WR, Bravo G, Parsons JC: The search for synergy: a critical review from a response surface perspective. Pharmacol Rev 1995, 47:331-385.

30. Tallarida RJ: Drug synergism: its detection and applications. J Pharmacol Exp Ther 2001, 298:865-872.

31. Loewe S: The problem of synergism and antagonism of combined drugs. Arzneimittelforschung 1953, 3:285-290.

32. Bliss Cl: The toxicity of poisons applied jointly. Ann Appl Biol 1939, 26:585-615.

33. Chou TC: Theoretical basis, experimental design, and computerized simulation of synergism and antagonism in drug combination studies. Pharmacol Rev 2006, 58:621-681.

34. Chou TC: Drug combination studies and their synergy quantification using the Chou-Talalay method. Cancer Res 2010, 70:440-446.

35. Chou TC, Talalay P: Quantitative analysis of dose-effect relationships: the combined effects of multiple drugs or enzyme inhibitors. Adv Enzyme Regul 1984, 22:27-55.

36. Martinez-Irujo JJ, Villahermosa ML, Alberdi E, Santiago E: A checkerboard method to evaluate interactions between drugs. Biochem Pharmacol 1996, 51:635-644.

37. Dahari $\mathrm{H}$, Ribeiro RM, Rice CM, Perelson AS: Mathematical modeling of subgenomic hepatitis $\mathrm{C}$ virus replication in Huh-7 cells. J Virol 2007, 81:750-760.

38. McLean AK, Luciani F, Tanaka MM: Trade-offs in resource allocation in the intracellular life-cycle of hepatitis C virus. J Theor Biol 2010, 267:565-572.

39. Nakabayashi J: A compartmentalization model of hepatitis $C$ virus replication: an appropriate distribution of HCV RNA for the effective replication. J Theor Biol 2012, 300:110-117.

40. Binder M, Sulaimanov N, Clausznitzer D, Schulze M, Hüber CM, Lenz SM, Schlöder JP, Trippler M, Bartenschlager R, Lohmann V, Kaderali L: Replication vesicles are load- and choke-points in the hepatitis C virus lifecycle. PLoS Pathog 2013, 9:e1003561.

41. Jonker DM, Visser SA, van der Graaf PH, Voskuyl RA, Danhof M: Towards a mechanism-based analysis of pharmacodynamic drug-drug interactions in vivo. Pharmacol Ther 2005, 106:1.

42. Kobayashi T, Koizumi Y, Takeuchi JS, Misawa N, Kimura Y, Morita S, Aihara K, Koyanagi Y, Iwami S, Sato K: Quantification of Deaminase Activity-Dependent and -Independent Restriction of HIV-1 Replication Mediated by APOBEC3F and APOBEC3G through Experimental-Mathematical Investigation. $J$ Virol 2014, 88:5881-5887.

43. Keith CT, Borisy AA, Stockwell BR: Multicomponent therapeutics for networked systems. Nat Rev Drug Discov $2005,4: 71-78$

44. Fitzgerald JB, Schoeberl B, Nielsen UB, Sorger PK: Systems biology and combination therapy in the quest for clinical efficacy. Nat Chem Biol 2006, 2:458-466.

45. Zimmermann GR, Lehár J, Keith CT: Multi-target therapeutics: when the whole is greater than the sum of the parts. Drug Discov Today 2007, 12:34-42.

46. Kirouac DC, Du JY, Lahdenranta J, Overland R, Yarar D, Paragas V, Pace E, McDonagh CF, Nielsen UB, Onsum MD: Computational modeling of ERBB2-amplified breast cancer identifies combined ErbB2/3 blockade as superior to the combination of MEK and AKT inhibitors. Sci Signal 2013, 6:ra68.

47. Lehár J, Stockwell BR, Giaever G, Nislow C: Combination chemical genetics. Nat Chem Biol 2008, 4:674-681.

48. Lehár J, Zimmermann GR, Krueger AS, Molnar RA, Ledell JT, Heilbut AM, Short GF, Giusti LC, Nolan GP, Magid OA, Lee MS, Borisy AA, Stockwell BR, Keith CT: Chemical combination effects predict connectivity in biological systems. Mol Syst Biol 2007, 3:80.

49. Owens CM, Mawhinney C, Grenier JM, Altmeyer R, Lee MS, Borisy AA, Lehár J, Johansen LM: Chemical combinations elucidate pathway interactions and regulation relevant to Hepatitis C replication. Mol Syst Biol 2010, 6:375.

50. Guedj J, Perelson AS: Second-phase hepatitis C virus RNA decline during telaprevir-based therapy increases with drug effectiveness: implications for treatment duration. Hepatology 2011, 53:1801-1808.

51. Guedj J, Neumann AU: Understanding hepatitis C viral dynamics with direct-acting antiviral agents due to the interplay between intracellular replication and cellular infection dynamics. J Theor Biol 2010, 267:330-340.

52. Guedj J, Dahari H, Rong L, Sansone ND, Nettles RE, Cotler SJ, Layden TJ, Uprichard SL, Perelson AS: Modeling shows that the NS5A inhibitor daclatasvir has two modes of action and yields a shorter estimate of the hepatitis C virus half-life. Proc Natl Acad Sci U S A 2013, 110:3991-3996.

53. Rong L, Guedj J, Dahari H, Coffield DJ, Levi M, Smith P, Perelson AS: Analysis of hepatitis C virus decline during treatment with the protease inhibitor danoprevir using a multiscale model. PLoS Comput Biol 2013, 9:e1002959.

54. Rong L, Perelson AS: Mathematical analysis of multiscale models for hepatitis C virus dynamics under therapy with direct-acting antiviral agents. Math Biosci 2013, 245:22-30

55. Rong L, Dahari H, Ribeiro RM, Perelson AS: Rapid emergence of protease inhibitor resistance in hepatitis $\mathrm{C}$ virus. Sci Trans/ Med 2010, 2:30ra32.

doi:10.1186/1742-4682-11-41

Cite this article as: Koizumi and Iwami: Mathematical modeling of multi-drugs therapy: a challenge for determining the optimal combinations of antiviral drugs. Theoretical Biology and Medical Modelling 2014 11:41. 